\title{
Day-ahead Resource Scheduling Including Demand Response for Electric Vehicles
}

\author{
Hugo Morais, Member, IEEE, João Soares, Tiago Sousa, Zita Vale, Senior Member, IEEE, \\ Pedro Faria, Student Member, IEEE
}

\begin{abstract}
Energy resource scheduling is becoming increasingly important, as the use of distributed resources is intensified and massive gridable vehicle (V2G) use is envisaged. This paper presents a methodology for day-ahead energy resource scheduling for smart grids considering intensive use of distributed generation and V2G.

The main focus of this paper is the comparison of different $\mathrm{EV}$ management approaches in the day-ahead energy resources management, namely uncontrolled charging, smart charging, V2G and Demand Response (DR) programs in the V2G approach. Three different DR programs are designed and tested in this paper (trip reduce, shifting reduce and reduce+shifting). Other important contribution of the paper is the comparison between deterministic and computational intelligence techniques to reduce execution time.

The proposed scheduling is solved with a modified particle swarm optimization. Mixed integer non-linear programming is also used for comparison purposes. Full ac power flow calculation is included to allow taking into account the network constraints.
\end{abstract}

A case study with a 33 bus distribution network and 2000 V2G resources is used to illustrate the performance of the proposed method.

Index Terms - Demand Response, Electric Vehicle, Energy Resource Management, Particle Swarm Optimization.

\section{NOMENCLATURE}

$\Delta t \quad$ Period $t$ duration (e.g. 15 min., 30 min., 1 hour...)

$\eta_{c(V)} \quad$ Grid-to-Vehicle efficiency when the vehicle $V$ is in

$\begin{array}{ll}\eta_{d(V)} & \text { Vharge mode } \\ & \text { Vehiscle-to-Grid efficiency when the vehicle } V \text { is in }\end{array}$

$\theta_{b} \quad$ Voltage angle at bus $b$ (rad)

$\theta_{b}^{\max } \quad$ Maximum voltage angle at bus $b(\mathrm{rad})$

$\theta_{b}^{\text {min }} \quad$ Minimum voltage angle at bus $b(\mathrm{rad})$

$\theta_{k} \quad$ Voltage angle at bus $k(\mathrm{rad})$

$B_{b k} \quad$ Imaginary part of the element in $y_{b k}$ corresponding to the $b$ row and $k$ column

$c_{\text {Charge }(V, t)} \quad$ Charge price of vehicle $V$ in period $t$

This work was supported by FEDER Funds through COMPETE program and by National Funds through FCT under the projects FCOMP-01-0124FEDER: PEst-OE/EEI/UI0760/2011, PTDC/EEA-EEL/099832/2008, and PTDC/SEN-ENR/099844/2008.

H. Morais, J. Soares, T. Sousa, Z. Vale, and P. Faria, are with GECAD Knowledge Engineering and Decision-Support Research centre of the School of Engineering - Polytechnic of Porto (ISEP/IPP), Dr. António Almeida, 431, 4200-072 Porto, Portugal (e-mail: \{japs, hgvm tas zav, pnf\}@isep.ipp.pt ).
$c_{D G(D G, t)}$
$c_{\text {Discharge }(V, t)}$
$c_{E A P(D G, t)}$
$c_{N S D(L, t)}$
$c_{\text {Shift }(V, t)}$
$c_{\text {Supplier }(S, t)}$
$c_{\text {TripRed }(V, t)}$
$E_{\text {BatCap }(V)}$
$E_{\text {MinCharge }(V, t)}$
$E_{\text {Stored }(V, t)}$
$E_{\text {Trip }(V, t)}$
$E_{\text {Trip Red }(V, t)}$
$E_{\text {TripRedMax }(V, t)}$
$G_{b k}$
$N_{b}$
$N_{D G}$
$N_{D G}^{b}$
$N_{L}$
$N_{L}^{b}$
$N_{S}$
$N_{S}^{b}$
$N_{V}$
$N_{V}^{b}$
$N_{V}^{b \_n o S h i f t}$
$N_{V}^{b-S h i f t}$
$P_{\text {Charge }(V, t)}$
$P_{\text {Charge }(V, t)}^{b}$
$P_{\text {Chargelimit }(V, t)}$
Generation price of $D G$ unit in period $t$
Discharge price of vehicle $V$ in period $t$
Excess available power price of $D G$ unit in period $t$
Non-supplied demand price of load $L$ in period $t$
Trip shifting price for vehicle $V$ in period $t$
Energy price of external supplier $S$ in period $t$
Trip reduction price contracted with vehicle $V$ in period $t$
Battery energy capacity of vehicle $V$
Minimum stored energy to be guaranteed at the end of period $t$, for vehicle $V$
Active energy stored in vehicle $V$ at the end of period $t$
Vehicle $V$ energy consumption in period $t$
Demand response energy reduction of vehicle trip $V$ in period $t$
Maximum energy reduction for vehicle $V$ trip in period $t$
Real part of the element in $y_{b k}$ corresponding to the $b$ row and $k$ column
Total number of buses
Total number of distributed generators
Total number of distributed generators at bus $b$
Total number of loads
Total number of loads at bus $b$
Total number of external suppliers
Total number of external suppliers at bus $b$
Total number of vehicles $V$
Total number of vehicles at bus $b$
Total number of vehicles at bus $b$ with original trips
Total number of vehicles at bus $b$ shifting their trips
Power charge of vehicle $V$ in period $t$
Power charge of vehicle $V$ at bus $b$ in period $t$
Maximum power charge of vehicle $V$ in period $t$ 
$P_{D G(D G, t)} \quad$ Active power generation of distributed generation

$P_{D G(D G, t)}^{b}$ Active power generation of distributed generation unit $D G$ at bus $b$ in period $t$

$P_{D G M a x L i m i t(D G, t)}$

$P_{D G M i n L i m i t(D G, t)}$

Maximum active power generation of distributed generator unit $D G$ in period $t$

Minimum active power generation of distributed generator unit $D G$ in period $t$

$P_{\text {Discharge }(V, t)}$

$P_{\text {Discharge }(V, t)}^{b}$

Power discharge of vehicle $V$ in period $t$

$P_{\text {DischargeLimit }(V, t)}$

Power discharge of vehicle $V$ at bus $b$ in period $t$

$P_{E A P(D G, t)}$

$P_{E A P(D G, t)}^{b}$

$P_{\text {Load }(L, t)}^{b}$

$P_{N S D(L, t)}$

$P_{N S D(L, t)}^{b}$

$P_{\text {Supplier }(S, t)}$

$P_{\text {Supplier }(S, t)}^{b}$

$P_{\text {SupplierLimit }(S, t)}$

$Q_{D G(D G, t)}^{b}$

$Q_{D G \operatorname{MaxLimit}(D G, t)}$

$Q_{D G M i n L i m i t}(D G, t)$

$Q_{\text {Load }(L, t)}^{b}$

$Q_{\text {Supplier }(S, t)}^{b}$

$Q_{\text {SupplierLimit }(S, t)}$

T

tLast

$S_{b k}^{\max }$

$V_{b(t)}$

$V_{b}^{\max }$

$V_{b}^{\min }$

$V_{k(t)}$

$X_{(V, t)}$

Maximum power discharge of vehicle $V$ in period $t$

Excess available power in $D G$ unit in period $t$

Excess available power in $D G$ unit at bus $b$ in period $t$

Active power demand of load $L$ at bus $b$ in period $t$

Non-supplied demand for load $L$ in period $t$

Non-supplied demand for load $L$ at bus $b$ in period $t$

Active power flow in the branch connecting to external supplier $S$ in period $t$

Active power flow in the branch connecting to upstream supplier $S$ at bus $b$ in period $t$

Maximum active power of upstream supplier $S$ in period $t$

Reactive power generation of distributed generation unit $D G$ at bus $b$ in period $t$

Maximum reactive power generation of distributed generator unit $D G$ in period $t$

Minimum reactive power generation of distributed generator unit $D G$ in period $t$

Reactive power demand of load $L$ at bus $b$ in period $t$

Reactive power flow in the branch connecting to upstream supplier $S$ at bus $b$ in period $t$

Maximum reactive power of upstream supplier $S$ in period $t$

Total number of periods

Last connected period of vehicle $V$ before

$E_{\text {Trip }(V, t)}$

Maximum apparent power flow established in line that connected bus $b$ and $k$

Voltage magnitude at bus $b$ in period $t$

Maximum voltage magnitude at bus $b$

Minimum voltage magnitude at bus $b$

Voltage magnitude at bus $k$ in period $t$

Binary variable of vehicle $V$ related to power discharge in period $t$

$X_{D G(D G, t)}$

$Y_{(V, t)}$

Binary decision variable of unit $D G$ in period $t$

Binary variable of vehicle $V$ related to power charge in period $t$

$y_{b k}$

Admittance of line that connect bus $b$ and $k$

$y_{\text {Shunt_b }}$

Shunt admittance of line connected to bus $b$

$Z_{(V)}$

Trip shifting decision binary variable

\section{INTRODUCTION}

The electrification of the transportation sector brings more challenges and offers new opportunities to network planning and operation [1]. Continued improvements of Electric Vehicles (EVs) envisage their massive use, therefore meaning that large quantities of EVs must be considered by future power systems, in terms of the required supply to ensure their users' daily travels [2]. In future scenarios of intensive EVs penetration, the typical load diagram can be significantly changed from the present one without EVs [2]. Smart grids on the other hand, can use V2Gs intelligently as distributed energy resources when the vehicles are parked. All of these adds further complexity to planning and operation of smart grids operation requiring new methods and more computational resources [2-4].

In that context with such complexity, computational intelligence methods are important to obtain solutions for large dimension problems in an acceptable time [4]. Authors in [5] present a unit commitment model with V2G using the Particle Swarm Optimization (PSO) to reduce costs and emissions in smart grids, this work attests the importance of using computational intelligence methods in many aspects of smart grid optimization. PSO is an effective method to determine the solution of large-scale nonlinear optimization problems [6].

Demand Response (DR) has already proven itself as a valuable tool to ensure reliability of the bulk electric system and is evolving and playing a great role in the electric industry [7-9]. For instance, during the summer heat wave of 2006, the Midwest ISO avoided firm load shed using interruptible load, demand-side management, and public appeals [9]. EVs have the possibility to provide a significant amount of DR through a variety of approaches while using their storage potential to enable a higher penetration of intermittent and variable generation such as wind and solar energy resources [9]. In this paper EVs DR program are proposed and consist in the reduction of the EVs trip distance and/or on trip time shifting, changing the initial travel requirements. Considering the available alternatives, the paper considers 6 scenarios for EVs:

- Uncontrolled charging

- Smart charging

- Vehicle-to-Grid

- EVs DR models proposed in this paper:

- Trip distance reduce

o Trip shifting

- Trip distance reduce and shifting together

The operator managing the network resources will remunerate the participation of EVs in the DR event, giving this way an incentive in order to reach both economic and technical objectives inherent to the network operation.

The proposed application uses a modified Particle Swarm Optimization (PSO) approach that considers dynamic changing of velocity limits [10]. This enables its use for addressing real world large-scale problems in shorter execution time than deterministic methods, providing system operators with adequate decision support and achieving efficient resource scheduling, even when a significant number of alternative scenarios should be considered $[2,10]$. 
The paper is organized as follows: After the initial introductory section, Section II explains the importance of V2G contracts and DR opportunities for EVs for energy resource management, in the scope of distribution systems, using the smart grid paradigm. Section III presents the problem formulation, including the resources and network constraints. Section IV presents the case studies using a 33 bus distribution network and considering 2000 vehicles. Section V presents the most important conclusions of the work.

\section{DEMAND RESPONSE FOR EVS IN SMART GRIDS}

This section explains the concepts used in the paper regarding EVs management and DR models in the context of smart grids. Two DR programs are described with more detail in this Section: trip reduce and trip shifting. The use of the proposed DR models can be activated every time that the price of the energy reaches a predefined value. Other potential use of these programs can be fruitful in the context of network management or ancillary services [7, 11]. DR program participation is voluntary [11].

Fig. 1 presents the EVs management strategies considered in this paper and the EVs DR proposed approaches. A brief description for each strategy is depicted in the figure, e.g. problem required constraints, as well as the output expected with the scheduling optimization.

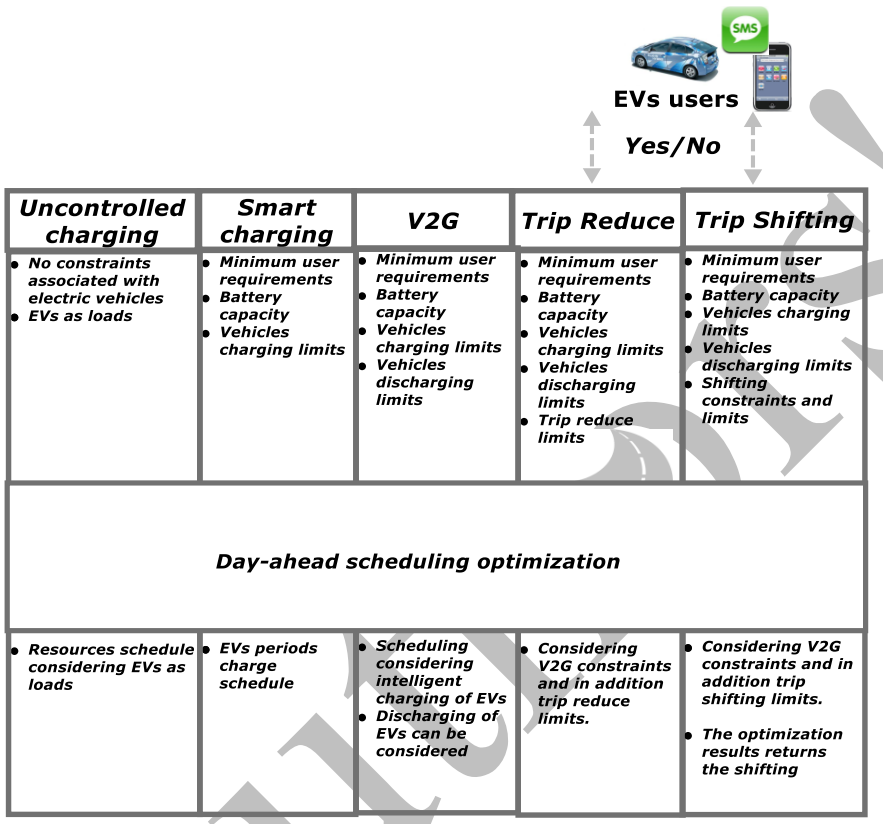

Fig. 1. EVs management and DR models considered

\section{A. Trip reduce demand response program}

The idea is to provide network operator with another useful resource that consists in reducing vehicles charging necessities. This demand response program enables vehicle users to get some profit by agreeing to reduce their travel necessities and minimum battery level requirements.

In phase 1 an initial optimization is made assuming that EVs which contracted DR option will participate. With the optimization results it is possible to identify which EVs users are scheduled to participate in the DR event. After that, these EV users can be invited to participate, e.g. through internet application, SMS message. The network operator should wait for a response within a time limit. With the responses of EVs, users the optimization program reschedules the day-ahead problem with the updated information. If additionally EV users are scheduled to participate in the DR program, according to the new optimization results, the operator should follow the same procedure. The users that do not respond within the time limit are excluded from the present DR event.

\section{B. Trip shifting demand response program}

In what concerns the trip shifting DR program for EVs it aims to provide another useful resource for the network operator. This demand response program enables vehicle users to provide a list of optional travelling periods for their expected travel trips. The program enables the network operator to shift EVs load by remunerating their users, reducing operational costs and alleviating network contingencies. The shifting is limited to the alternatives that users impose, restraining the computational execution time of optimization process at the same time. Phase 1 consist of considering users' alternative trips in the optimization model. After this step, the network operator can acknowledge EVs users with shifting results from optimization phase 1 to know if they are able to participate in the next day. The acknowledgment of users' participation (phase 2) in the program is important for network operator in order to obtain the appropriate resources scheduling and reduce operational costs.

\section{ENERGy Resource SCHEdULING Formulation}

This section presents the mathematical formulation of the proposed methodology in this paper. The Particle Swarm Optimization (PSO) approach is also presented in this section.

\section{A. Problem formulation}

This methodology is used to support network operator to obtain an adequate energy resource management for the next day, including Electric Vehicles (EVs) resource, in the smart grid context. In terms of problem description, the network operator has contracts for managing the resources installed in the grid, including load demand. The load demand can be satisfied by the distributed generation resources, by the discharge of EVs, and by external suppliers (namely retailers, the electricity pool). The use of Vehicle-to-Grid (V2G) discharge, and the respective charge, considers $\mathrm{V} 2 \mathrm{G}$ user profiles and requirements. The network influence is included in this methodology, through ac power flow calculation, voltage limits and line thermal limits.

The energy resource scheduling problem is a Mixed Integer Non-Linear Programming (MINLP) problem. The objective function aggregates all the costs with the energy resources. The energy resource model includes: distributed generation, energy acquisition to external suppliers, the V2G discharge or charge energy, the non-supplied demand, the excess available power $[2,3]$, trip reduce demand response and trip shifting demand response model for EVs. The present problem differs 
from previous works $[2,3]$ by including network constraints, which are important in real world operation, and by including the EVs demand response programs.

In order to achieve a good scheduling of the available energy resources, it is necessary to apply a multi-period optimization; the presented formulation is generic for a specified time period (from period $t=1$ to $t=T$ ) [2,11]. The model includes an ac power flow algorithm that allows network constraints to be considered, leading to a Mixed Integer Non-Linear Programming (MINLP) problem.

\section{Minimize}

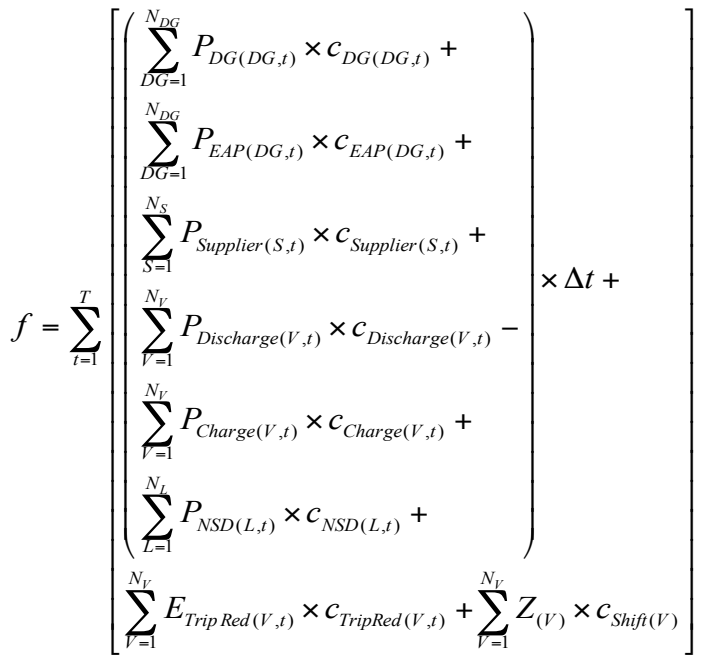

The objective function considers $\Delta t$ to allow different period $t$ duration. For instance, for 30 minutes period $t$ duration, the value of $\Delta t$ should be 0.5 if the costs function are specified in an hour basis.

In order to improve the solution feasibility the mathematical model includes variables concerning the excess available power $\left(P_{E A P(D G, t)}\right)$ and non-supplied demand $\left(P_{N S D(L, t)}\right) \cdot P_{E A P(D G, t)}$ is important because the network operator can establish contracts with uninterruptible generation ("take or pay" contracts) with, for instance, producers based on renewable energy sources. In extreme cases, when the load is lower than uninterruptible generation the value of $P_{E A P(D G, t)}$ is different from zero. $P_{N S D(L, t)}$ is positive when the available resources are not enough to satisfy load demand.

The minimization of objective function (1) is subject to the following constraints:

- The network active (2) and reactive (3) power balance with power loss in each period $t$ :

$$
\begin{aligned}
& \sum_{D G=1}^{N_{D G}^{b}}\left(P_{D G(D G, t)}^{b}-P_{E A P(D G, t)}^{b}\right)+\sum_{S=1}^{N_{S}^{b}} P_{\text {Supplier }(S, t)}^{b}+ \\
& \sum_{L=1}^{N_{L}^{b}}\left(P_{N S D(L, t)}^{b}-P_{\text {Load }(L, t)}^{b}\right)+\sum_{V=1}^{N_{V}^{b}}\left(P_{\text {Discharge }(V, t)}^{b}-P_{\text {Charge }(V, t)}^{b}\right)= \\
& \sum_{k=1}^{N_{B}} V_{b(t)} \times V_{k(t)}\left(G_{b k} \cos \left(\theta_{b(t)}-\theta_{k(t)}\right)+B_{b k} \sin \left(\theta_{b(t)}-\theta_{k(t)}\right)\right) \\
& \forall t \in\{1, . ., T\} ; k \neq b ; N_{V}^{b}=N_{V}^{b-n o S h i f t}+N_{V_{-}^{b} \text { Shift }} \times Z_{(V, t)}
\end{aligned}
$$

$$
\begin{aligned}
& \sum_{D G=1}^{N_{D G}^{b}} Q_{D G(D G, t)}^{b}+\sum_{S=1}^{N_{S}^{b}} Q_{\text {Supplier }(S, t)}^{b}-\sum_{L=1}^{N_{L}^{b}} Q_{\text {Load }(L, t)}^{b}= \\
& \sum_{k=1}^{N_{B}} V_{b(t)} \times V_{k(t)}\left(G_{b k} \sin \left(\theta_{b(t)}-\theta_{k(t)}\right)-B_{b k} \cos \left(\theta_{b(t)}-\theta_{k(t)}\right)\right) \\
& \forall t \in\{1, . ., T\} ; k \neq b
\end{aligned}
$$

- Bus voltage magnitude and angle limits:

$V_{b}^{\min } \leq V_{b(t)} \leq V_{b}^{\max } \forall t \in\{1, \ldots, T\}$

$\theta_{b}^{\min } \leq \theta_{b(t)} \leq \theta_{b}^{\max } \forall t \in\{1, \ldots, T\}$

- Line thermal limits:

$\left|V_{b(t)} \times\left(\left[\left(V_{b(t)}-V_{k(t)}\right) y_{b k}\right]^{*}+\left[V_{b(t)} \times \frac{1}{2} y_{\text {Shunt_b}}\right]^{*}\right)\right| \leq S_{b k}^{\max }$

$\forall t \in\{1, . ., T\}$

- Maximum distributed generation limit in each period $t$ :

$P_{D G(D G, t)} \leq X_{D G(D G, t)} \times P_{D G M a x L i m i t(D G, t)}$

$P_{D G(D G, t)} \geq X_{D G(D G, t)} \times P_{D G M i n L i m i t(D G, t)}$

$\forall t \in\{1, \ldots, T\} ; \forall D G \in\left\{1, \ldots, N_{D G}\right\}$

$Q_{D G(D G, t)} \leq X_{D G(D G, t)} \times Q_{D G \operatorname{MaxLimit}(D G, t)}$

$Q_{D G(D G, t)} \geq X_{D G(D G, t)} \times Q_{D G \operatorname{MinLimit}(D G, t)}$

$\forall t \in\{1, \ldots, T\} ; \forall D G \in\left\{1, \ldots, N_{D G}\right\}$

- Upstream supplier maximum limit in each period $t$ :

$P_{\text {Supplier }(S, t)} \leq P_{\text {SupplierLimit }(S, t)}$

$\forall t \in\{1, \ldots, T\} ; \forall S \in\left\{1, \ldots, N_{S}\right\}$

$Q_{\text {Supplier }(S, t)} \leq Q_{\text {SupplierLimit }(S, t)}$

$\forall t \in\{1, \ldots, T\} ; \forall S \in\left\{1, \ldots, N_{S}\right\}$

- Vehicle technical limits in each period $t$ :

- The vehicle charge and discharge are not simultaneous:

$X_{(V, t)}+Y_{(V, t)} \leq 1$

$\forall t \in\{1, \ldots, T\} ; \forall V \in\left\{1, \ldots, N_{V}\right\} ; X_{(V, t)}$ and $Y_{(V, t)} \in\{0,1\}$

- Battery balance for each vehicle. The energy consumption for period $\mathrm{t}$ travel has to be considered jointly with the energy remaining from the previous period and the charge/discharge in the period:

$E_{\text {Stored }(V, t)}=E_{\text {Stored }(V, t-1)}+\eta_{c(V)} \times P_{\text {Charge }(V, t)} \times \Delta t$

$E_{\text {Trip }(V, t)}-\frac{1}{\eta_{d(v)}} \times P_{\text {Discharge }(V, t)} \times \Delta t$

$\forall t \in\{1, \ldots, T\} ; \forall V \in\left\{1, \ldots, N_{V}\right\} ; E_{\operatorname{Trip}(V, t)}=P_{\operatorname{Trip}(V, t)} \times \Delta t ;$

- Discharge limit for each vehicle considering the battery discharge rate:

$P_{\text {Discharge }(V, t)} \leq P_{\text {DischargeLimit }(V, t)} \times X_{(V, t)}$

$\forall t \in\{1, \ldots, T\} ; \forall V \in\left\{1, \ldots, N_{v}\right\} ; X_{(V, t)} \in\{0,1\}$ 
- Charge limit for each vehicle considering the battery charge rate:

$P_{\text {Charge }(V, t)} \leq P_{\text {Chargelimit }(V, t)} \times Y_{(V, t)}$

$\forall t \in\{1, \ldots, T\} ; \forall V \in\left\{1, \ldots, N_{v}\right\} ; Y_{(V, t)} \in\{0,1\}$

- Vehicle battery discharge limit considering the battery balance:

$$
\begin{aligned}
& \frac{1}{\eta_{d(V)}} \times P_{\text {Discharge }(V, t)} \times \Delta t \leq E_{\text {Stored }(V, t-1)} \\
& \forall t \in\{1, \ldots, T\} ; \quad \forall V \in\left\{1, \ldots, N_{V}\right\} ; \Delta t=1 ;
\end{aligned}
$$

- Vehicle battery charge limit considering the battery capacity and previous charge status:

$$
\begin{aligned}
& \eta_{c(V)} \times P_{\text {Charge }(V, t)} \times \Delta t \leq E_{\text {BatCap }(V)}-E_{\operatorname{Stored}(V, t-1)} \\
& \forall t \in\{1, \ldots, T\} ; \forall V \in\left\{1, \ldots, N_{V}\right\}
\end{aligned}
$$

- Battery capacity limit for each vehicle:

$E_{\text {Stored }(V, t)} \leq E_{\text {BatCap }(V)} \forall t \in\{1, \ldots, T\} ; \forall V \in\left\{1, \ldots, N_{V}\right\}$

- Minimum stored energy to be guaranteed at the end of period $t$. This can be seen as a reserve energy (fixed by the EVs users) that can be used for a regular travel or a unexpected travel in each period:

$$
\begin{aligned}
& E_{\text {Stored }(V, t)} \geq E_{\text {MinCharge }(V, t)}-E_{\text {TripRed }(V, t)} \\
& E_{\text {Mincharge }(V, \text { tLast })} \geq E_{\text {Trip }(V, t)} \forall t \in\{1, \ldots, T\} ; \forall V \in\left\{1, \ldots, N_{V}\right\} \\
& E_{\text {TripRed }(V, t)} \leq E_{\text {TripRedMax }(V, t)} \quad \forall t \in\{1, \ldots, T\} ; \forall V \in\left\{1, \ldots, N_{V}\right\}
\end{aligned}
$$

\section{B. Particle swarm approach}

The PSO concept began as a simulation of simple social systems like the flocks of birds or the schools of fish [12]. The main advantage of PSO is its simplicity, while being capable of delivering accurate results in a consistent manner. It is fast and also very flexible, being applicable to a wide range of problems, with limited computational requirements [6]. The original PSO relies on fixed velocity limits that are not changed during the swarm search process (PSO iterations) $[12,13]$. Research work performed by Fan and Shi $[12,14]$ has shown that an appropriate dynamic change of maximum velocities can improve the performance of the PSO algorithm.

In the present implementation to the problem of day-ahead scheduling, maximum and minimum values of velocity limits can change dynamically through the search process. The initial velocities are set for each variable according to its type, e.g. maximum velocities for generators reactive variables are set to 0.02 while minimum velocities are set to -0.01 . Maximum and minimum velocities for generators active power are calculated by means of a rank algorithm that takes into account the generators energy price.

In the evaluation phase the implemented mechanism will check for constraint violations, namely bus lower and overvoltage violations (4-5) and line thermal limits (6). If there is any violation of the above constraints the algorithm will mark the variables that can possibly help to alleviate these violations. In the case of bus lower voltage violations, the mechanism will mark DG reactive power and V2G resources variables, to increase reactive power and discharges, respectively. In the case of bus overvoltage violations, the mechanism will mark DG reactive power variables to decrease and nearby EVs to charge. The buses selected to get the appropriate $\mathrm{V} 2 \mathrm{G}$ and DG resources are the buses where violations occurred as well as the buses that were preceding it.

Line thermal limit violations can be corrected in two ways: reducing $\mathrm{V} 2 \mathrm{G}$ charge or increasing generation in the downstream lines. The mechanism marks $\mathrm{V} 2 \mathrm{G}$ charge to be reduced and DG generation production to be increased. More information about voltage drop in radial distribution networks can be found in [15].

The velocity limits of the marked variables are changed according to the type of signaling. For instance, when DG reactive power variables are marked, the maximum velocities of these variables are increased by $20 \%$. When the DG reactive power variables are marked to decrease, the minimum velocities of these variables are decreased by $20 \%$.

The described mechanism contributes to a faster convergence to a solution without violations, as well as improving the solution fitness. To improve fitness function the mechanism works as follows:

- It tries to increase V2G charge variables values when V2G charge price is lower than mean generation cost acting on maximum velocity limits of corresponding variables;

- It tries to increase $\mathrm{V} 2 \mathrm{G}$ discharge variables values when V2G discharge price is lower than mean generation cost acting on minimum velocity limits of corresponding variables;

- It tries to apply DR V2G trip reduce program (when available) by increasing corresponding variables when DR program price is lower than the sum of mean generation cost and the respective vehicle charge price.

Looking at the problem formulation presented in Section A, namely the objective function, it can be seen why the above aspects improve the solution. The variables of DR trip shifting program are not controlled by the described mechanism.

The initial swarm population is randomly generated between the upper and the lower bounds of variables, except from V2G variables that are initialized with zeros. During swarm search the algorithm checks whether to charge or discharge vehicles as well as to apply DR trip reduce programs as needed or advantageous. DR shifting variables are randomly initialized by the swarm.

A robust power flow model from [16] is included in the modified PSO approach to check solutions feasibility during swarm search process. The load system balance (2-3) is validated by a power flow algorithm, and the power losses are compensated by the energy suppliers or DG generators. Vehicle battery balance constraints (12) are checked before fitness evaluation. If the values from swarm solutions are not according to the constraint limits, the solution is corrected by direct repair method. Direct repair method can be used instead of indirect repair method such as penalty factors providing an efficient way of correcting solutions before evaluating the 
fitness function [17].

\section{CASE-STUdY}

This section presents the case study used in this paper to illustrate the proposed models. For that, an exact method, namely Mixed Integer Non-Linear Programming (MINLP) obtained using the software GAMS, is compared with the PSO methodology in terms of execution time and solution quality.

This case study considers a 33 bus distribution network as can be found in [2].

Table I presents the values of prices for each resource, minimum and maximum available capacity during the day, and the number of units for each type of technology. The respective values for the ten considered suppliers, connected to the network in the substation, are also presented. Only the linear component of the sources cost functions is considered in this case study. The cost of EV discharge is low because as can be seen in the objective function (1) (see Section III), the discharge is considered a profit for EV users. The EV charge and loads cost is considered 0 (m.u./kWh) in this case study.

TABLE I

SOURCES CHARACTERIZATION

\begin{tabular}{l|c|c|c|c|c}
\hline \hline Resource & $\begin{array}{c}\text { Min. price } \\
(\mathrm{m} . \mathrm{u} . / \mathrm{kWh})\end{array}$ & $\begin{array}{c}\text { Max. price } \\
(\mathrm{m} . \mathrm{u} . / \mathrm{kWh})\end{array}$ & $\begin{array}{c}\text { Min. } \\
\text { capacity } \\
(\mathrm{kW})\end{array}$ & $\begin{array}{c}\text { Max. } \\
\text { capacity } \\
(\mathrm{kW})\end{array}$ & $\begin{array}{c}\text { Units } \\
\#\end{array}$ \\
\hline PV & 0.110 & 0.254 & 0 & 1,320 & 32 \\
\hline Wind & 0.060 & 0.136 & 255 & 505 & 5 \\
\hline CHP & 0.057 & 0.105 & 725 & 725 & 15 \\
\hline Biomass & 0.136 & 0.186 & 350 & 350 & 3 \\
\hline MSW & 0.076 & 0.102 & 210 & 210 & 2 \\
\hline Hydro & 0.059 & 0.095 & 80 & 80 & 2 \\
\hline Fuel cell & 0.115 & 0.180 & 240 & 240 & 7 \\
\hline Suppliers & 0.075 & 0.188 & 3,350 & 3,350 & 10 \\
\hline EV discharge & 0.025 & 0.025 & $8,625 *$ & 9,877 & 2,000 \\
\hline \multicolumn{7}{|c|}{ Total } \\
\hline \hline
\end{tabular}

The paper presents the results for five scenarios using 2000 EVs. This number is adequate for the dimension of the given MV distribution network under study considering high penetration of EVs in 2040. Uncontrolled charging is not presented in the case study because the optimal solution with 2000 EVs is not found (unfeasible due to network constraints). The DR scenarios consider the phase 1 of the described approach in Section II.

Regarding the parameterization of PSO approach, the number of iterations is set to 50 for each scenario. The parameters definition of PSO can be seen in table II. Gaussian mutation weights is used for mutation of the strategic parameters of PSO particles movement equation [18].

$$
\text { TABLE II }
$$

PARAMETERS OF PSO METHODS

\begin{tabular}{l|c}
\hline \multicolumn{2}{c}{ PARAMETERS OF PSO METHODS } \\
\hline \hline Parameters & Description \\
\hline Number of particles & 10 \\
\hline Inertia Weight & \multicolumn{1}{|c}{ Gaussian mutation weights } \\
\hline $\begin{array}{l}\text { Acceleration Coefficient } \\
\text { Best Position }\end{array}$ & $\begin{array}{l}\text { Randomly generated between the upper and } \\
\text { lower bounds, except from V2G variables. }\end{array}$ \\
\hline Cooperation Coefficient & 50 iterations \\
\cline { 1 - 2 } Initial swarm population & Equal to the upper bound of variables \\
\hline Stopping Criteria & Equal to the lower bound of variables \\
\hline Max. Positions & \\
\hline Min. Positions & \\
\hline \hline
\end{tabular}

Fig. 2 and 3 show the load and the EVs charge for the scenario using both trip reduce and shifting DR programs. Fig. 2 presents the results for the GAMS approach and Fig. 3 depicts the results for the PSO approach. The results are similar; however, PSO presents more discharging of EVs in load diagram peaks whereas GAMS schedules vehicles to charge during night hours and minimizing the impact during the day.

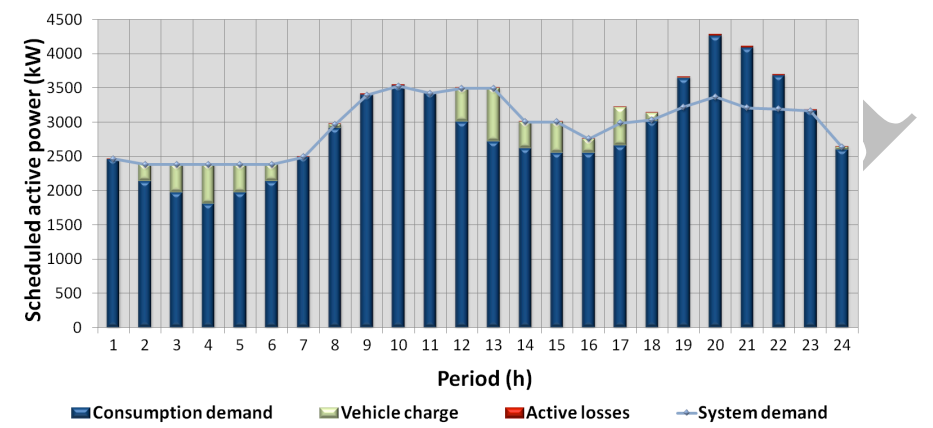

Fig. 2. Load and EVs charge and discharge profile of GAMS methodology.

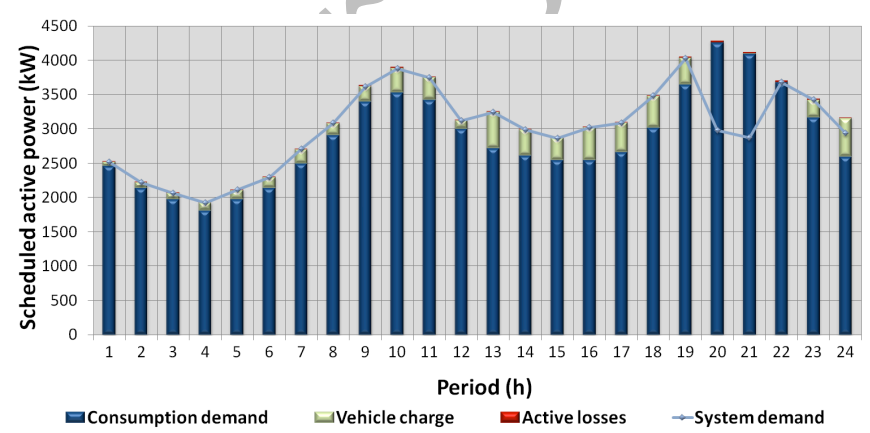

Fig. 3. Load and EVs charge and discharge profile of PSO methodology.

Table III presents the summary of the results for GAMS and the PSO approach for the five scenarios. In this case study PSO is approximately 2,700 times faster than GAMS methodology and the objective function is close to its cost in the five scenarios. The solutions for the scenarios presented in the paper for the PSO approach are selected arbitrary from a 200 trials run, thus not representing the best neither the worst case of those trials but aiming to show an random run average case. GAMS execution time is high and uses more than 24 hours to solve the optimization problem. This execution time is expected to rise exponentially with the increase of the number of resources and the complexity of the opportunities used in EVs such as DR programs. To note that this case study presents a modest 33 bus network size and 2,000 V2G resources. The execution time of GAMS approach is high, which is prohibitive for the day-ahead optimal resource scheduling. It can be desirable to test several forecasting scenarios and different renewable energy resources availability such as wind power. With the inclusion of V2G resources the number of forecasting scenarios will certainly increase.

TABLE III RESULTS OF THE MINLP AND PSO APPROACHES

\begin{tabular}{ccccccc}
\hline \hline & \multicolumn{2}{c}{ Operation cost (m.u.) } & \multirow{2}{*}{$\begin{array}{c}\text { Problem } \\
\text { variables }\end{array}$} & \multicolumn{2}{c}{ Execution time (s) } \\
& GAMS & PSO & & GAMS & PSO* $^{*}$ \\
\hline $\begin{array}{c}\text { Smart } \\
\text { Charging }\end{array}$ & $8,350.23$ & $8,408.68$ & 53,472 & 85,475 & 30.99 \\
\hline V2G & $8,177.47$ & $8,226.12$ & 197,472 & 89,748 & 31.98 \\
\hline Trip Shifting & $8,165.03$ & $8,190.59$ & 199,472 & 88,657 & 32.14 \\
\hline
\end{tabular}




\begin{tabular}{cccccc}
\hline Trip Reduce & $7,627.97$ & $7,887.03$ & 245,472 & 95,657 & 33.45 \\
\hline $\begin{array}{c}\text { Trip Reduce } \\
\text { + Shifting }\end{array}$ & $7,584.63$ & $7,750.77$ & 293,472 & 97,416 & 34.57 \\
\hline \hline
\end{tabular}

*average values obtained with 200 trials

Fig. 4 shows the objective function of 200 trials using the PSO approach for the $\mathrm{V} 2 \mathrm{G}$ scenario. The maximum objective function cost in 200 trials is approximately 8,340 m.u. and the minimum is approximately $8,180 \mathrm{~m}$.u. with an average value of 8,252 m.u. The variability of PSO approach can be considered low. A random trial for the given case study will fall into these values with high chance.

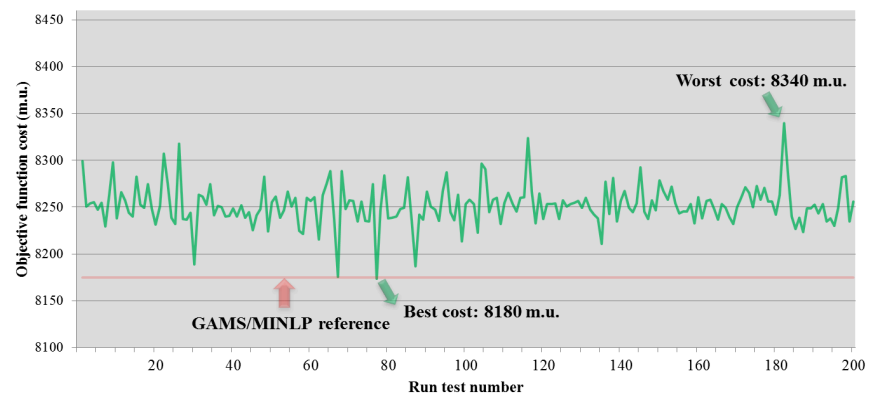

Fig. 4. Objective function cost for 200 trials using the PSO approach.

Fig. 5 show the energy resource scheduling for the five scenarios in the case study. In these figures, it is presented the results for the MINLP and PSO approach. Fig. 6 shows the charge and discharge profile obtained for the five scenarios as well. PSO approach uses more charging than GAMS approach except from V2G scenario. This happens due to PSO's stochasticity nature whereas GAMS is more accurate and thus providing solutions with lower cost.
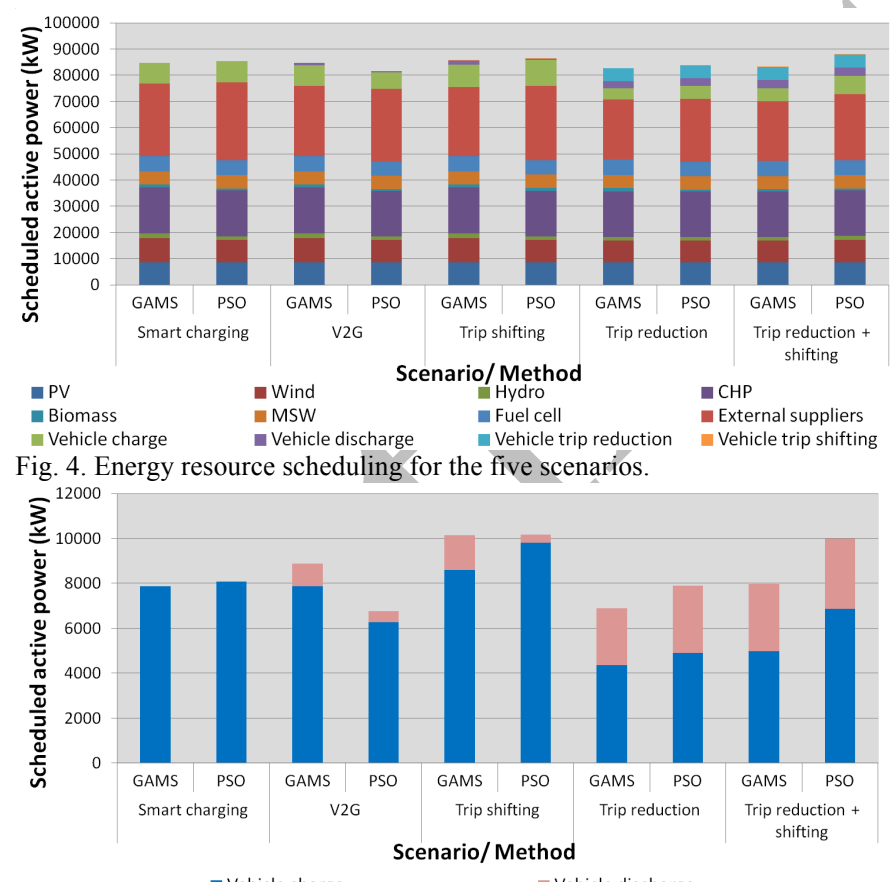

n Vehicle charge $\quad$ Vehicle discharge

Fig. 5. Charge and discharge scheduling for the five scenarios.

Fig. 6 depicts the trips energy consumption for DR trip reduce DR scenario. For the reduce program all the vehicles can participate reducing at most $50 \%$ of the needs of their travels. In this scenario almost all vehicles reduce their trips according to optimization results.

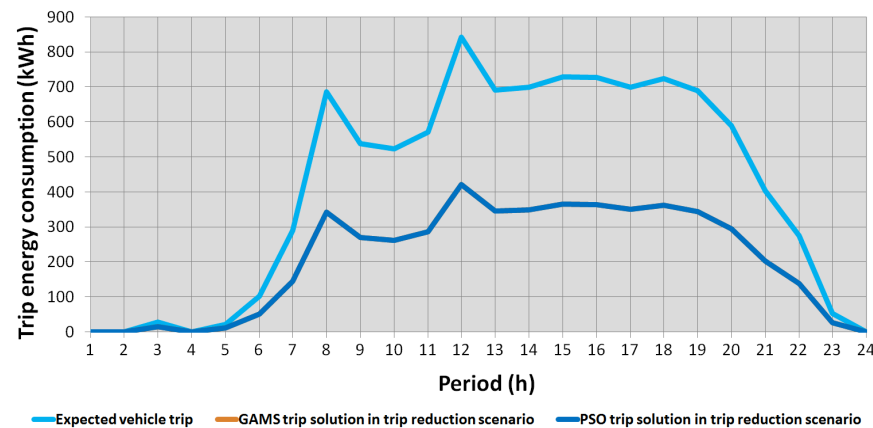

Fig. 6. Charge and discharge profile for the trip shift scenario.

Fig. 7 concerns the trip shifting program results. This scenario considered at most 200 vehicles to participate between periods 17-20. In the optimization solution, using this program, the resulting trip of $110 \mathrm{EVs}$ are dislocated from the initial forecasted trip. The decision of network operator prevails in order to use the DR programs.

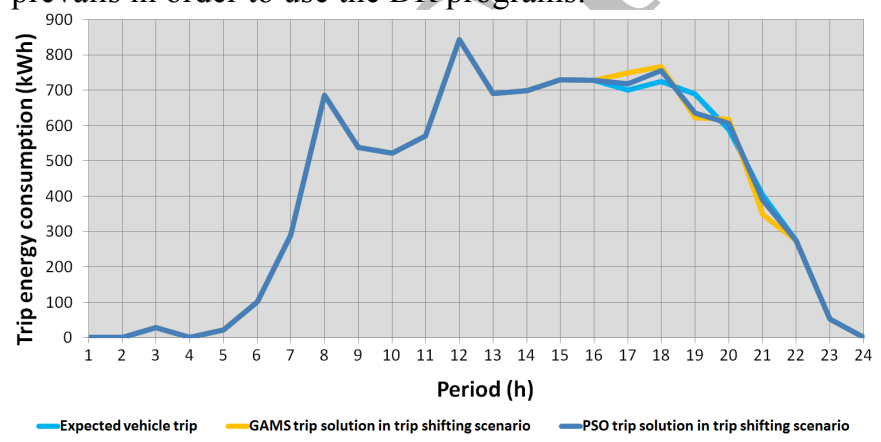

Fig. 7. Charge and discharge profile for the trip shift scenario.

\section{CONCLUSION}

The demand response for Electric Vehicles (EVs) users, in the context of smart grid, has been proposed in this paper considering the day-ahead optimal scheduling. A trip distance reduce and a trip shifting demand response (DR) programs has been designed and implemented in this paper. It was demonstrated the effectiveness of the proposed DR programs regarding the reduction of the operation costs in the point of view of the network operator.

The large amount of energy resources, including EVs, leads to an increase in the complexity of operation and planning of distribution networks. In this field, computational intelligence methods have an important role in the smart grid environment, where the traditional optimization techniques need high execution times to deliver a solution. Particle Swarm Optimization approach (PSO) with integrated ac power flow has been applied to each case study scenario.

When compared with the reference technique, MINLP in GAMS, in terms of execution time, the PSO approach is 2,700 times faster. The meta-heuristic presents low variability of the results and provides satisfactory solutions with reasonable execution time for the day-ahead problem context. GAMS provides the best solution quality albeit with high execution time which for the given context can be considered a useless solution. 


\section{REFERENCES}

[1]

K. Clement-Nyns, E. Haesen, and J. Driesen, "The Impact of Charging Plug-In Hybrid Electric Vehicles on a Residential Distribution Grid," Ieee Transactions on Power Systems, vol. 25, pp. 371-380, Feb 2010.

[2] T. Sousa, H. Morais, Z. Vale, P. Faria, and J. Soares, "Intelligent Energy Resource Management Considering Vehicle-to-Grid: A Simulated Annealing Approach," IEEE Transaction on Smart Grid, Special Issue on Transportation Electrification and Vehicleto-Grid Applications., 2012.

[3] J. Soares, T. Sousa, H. Morais, Z. Vale, and P. Faria, "An Optimal Scheduling Problem in Distribution Networks Considering V2G," in IEEE SSCI Symposium on Computational Intelligence Applications in Smart Grid (CIASG) Paris, France, 2011.

[4] G. K. Venayagamoorthy, "Dynamic, Stochastic, Computational and Scalable Technologies for Smart Grids," Ieee Computational Intelligence Magazine, vol. 6, pp. 22-35, Aug 2011.

[5] A. Y. Saber and G. K. Venayagamoorthy, "Plug-in Vehicles and Renewable Energy Sources for Cost and Emission Reductions," Ieee Transactions on Industrial Electronics, vol. 58, pp. 12291238, Apr 2011.

[6] Y. del Valle, G. K. Venayagamoorthy, S. Mohagheghi, J. C. Hernandez, and R. G. Harley, "Particle swarm optimization: Basic concepts, variants and applications in power systems," Ieee Transactions on Evolutionary Computation, vol. 12, pp. 171-195, Apr 2008.

[7] J. Medina, N. Muller, and I. Roytelman, "Demand Response and Distribution Grid Operations: Opportunities and Challenges," Smart Grid, IEEE Transactions on, vol. 1, pp. 193-198, 2010.

[8] A. J. Conejo, J. M. Morales, and L. Baringo, "Real-Time Demand Response Model," Smart Grid, IEEE Transactions on, vol. 1, pp. 236-242, 2010.

[9] M. Mallette and G. Venkataramanan, "The Role of Plug-In Hybrid Electric Vehicles in Demand Response and Beyond," 2010 Ieee Pes Transmission and Distribution Conference and Exposition: Smar Solutions for a Changing World, 2010.

[10] J. Soares, "Modified Particle Swarm Optimization for Day-Ahead Distributed Energy Resources Scheduling Including Vehicle-toGrid," MSc Thesis, School of Engineering - Polytechnic Institute of Porto, Porto, 2011.

[11] H. Morais, P. Kadar, P. Faria, Z. A. Vale, and H. M. Khodr, "Optimal scheduling of a renewable micro-grid in an isolated load area using mixed-integer linear programming," Renewable Energy, vol. 35, pp. 151-156, Jan 2010

[12] J. Kennedy and R. Eberhart, "Particle swarm optimization," 1995 Ieee International Conference on Neural Networks Proceedings, Vols 1-6, pp. 1942-1948, 1995.

[13] K. Y. Lee and M. A. El-Sharkawi, Modern heuristic optimization techniques : theory and applications to power systems. Piscataway, N.J.: IEEE Press ; Hoboken, N.J. : Wiley-Interscience, 2008.

[14] H. Fan and Y. Shi, "Study on Vmax of particle swarm optimization," in Proc. Workshop on Particle Swarm Optimization, Purdue School of Engineering and Technology, Indianapolis, IN, Apr. 2001.

[15] C. J. Dent, L.F. Ochoa, and G. P. Harrison, "Network Distributed Generation Capacity Analysis Using OPF With Voltage Step Constraints," Ieee Transactions on Power Systems, vol. 25, pp. 296-304, Feb 2010

[16] D. Thukaram, H. M. W. Banda, and J. Jerome, "A robust three phase power flow algorithm for radial distribution systems," Electric Power Systems Research, vol. 50, pp. 227-236, Jun 1 1999.

[17] Z. Michalewicz, "A survey of constraint handling techniques in evolutionary computation methods," Evolutionary Programming IV, pp. 135-155, 1995.

[18] R. A. Krohling, "Gaussian particle swarm with jumps," 2005 Ieee Congress on Evolutionary Computation, Vols 1-3, Proceedings, pp. 1226-1231, 2005.

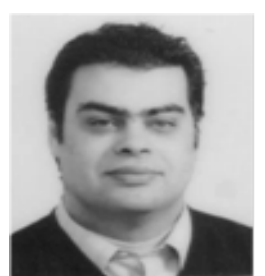

\section{BIOGRAPHIES}

Hugo Morais (M'11 S'08) received the BSC and Master degrees in Electrical Engineering from the Polytechnic Institute of Porto (ISEP/IPP), Portugal in 2005 and 2010 respectively. He is a Researcher at GECAD - Knowledge Engineering and DecisionSupport Research Center and a PhD student. His research interests include smart grids, virtual power players, and electricity markets.

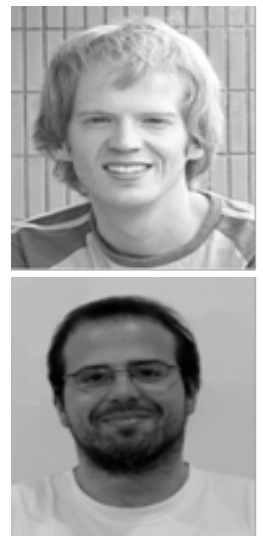

João Soares received the BSc and Master degrees in Electrical Engineering from the Polytechnic Institute of Porto (ISEP/IPP), Portugal in 2008 and 2011 respectively. $\mathrm{He}$ is an Assistant Researcher at GECAD - Knowledge Engineering and DecisionSupport Research Center. His research interests include heuristic optimization in power and energy systems.

Tiago Sousa received the BSc and Master degrees in Electrical Engineering from the Polytechnic Institute of Porto (ISEP/IPP), Portugal in 2009 and 2011 respectively. $\mathrm{He}$ is an Assistant Researcher at GECAD - Knowledge Engineering and DecisionSupport Research Center and a PhD student. His research interests include smart grids and heuristic optimization in power and energy systems.

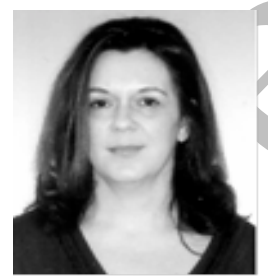

Zita A. Vale (SM'10 M'93 S'86) is the director of the Knowledge Engineering and Decision Support Research Center (GECAD) and a professor at the Polytechnic Institute of Porto.

She received her diploma in Electrical Engineering in 1986 and her PhD in 1993, both from University of Porto. Her main research interests concern Artificial Intelligence (A.I.) applications to Power System operation and control, Electricity Markets, Distributed Generation, and Smart Grids.

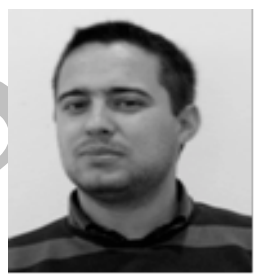

Pedro Faria ( $\left.\mathbf{S}^{\prime} \mathbf{1 0}\right)$ received the BSc and Master degrees in Electrical Engineering from the Polytechnic Institute of Porto (ISEP/IPP), Portugal in 2008 and 2011 respectively. He is an Assistant Researcher at GECAD - Knowledge Engineering and Decision-Support Research Center and a PhD student. His research interests include demand response, smart grids, and electricity markets. 